\title{
Variability of Gamma\#Ray Burst Afterglows due to Interstellar Turbulence
}

\section{Citation}

Wang, Xiaohu, and Abraham Loeb. 2000. "Variability of Gamma\#Ray Burst Afterglows

due to Interstellar Turbulence." The Astrophysical Journal 535 (2): 788-97. https://

doi.org/10.1086/308888.

\section{Permanent link}

http://nrs.harvard.edu/urn-3:HUL.InstRepos:41393274

\section{Terms of Use}

This article was downloaded from Harvard University's DASH repository, and is made available under the terms and conditions applicable to Other Posted Material, as set forth at http:// nrs.harvard.edu/urn-3:HUL.InstRepos:dash.current.terms-of-use\#LAA

\section{Share Your Story}

The Harvard community has made this article openly available.

Please share how this access benefits you. Submit a story.

Accessibility 
THE AsTROPHYSICAL JOURNAL, 535:788-797, 2000 June 1

(C) 2000. The American Astronomical Society. All rights reserved. Printed in U.S.A.

\title{
VARIABILITY OF GAMMA-RAY BURST AFTERGLOWS DUE TO INTERSTELLAR TURBULENCE
}

\author{
Xiaohu Wang and Abraham Loeb \\ Harvard-Smithsonian Center for Astrophysics, 60 Garden Street, Cambridge, MA 02138 \\ Received 1999 October 26; accepted 2000 January 20
}

\begin{abstract}
Gamma-ray burst (GRB) afterglows are commonly interpreted as synchrotron emission from a relativistic blast wave produced by a point explosion in an ambient medium, plausibly the interstellar medium of galaxies. We calculate the amplitude of flux fluctuations in the light curves of afterglows due to inhomogeneities in the surrounding medium. Such inhomogeneities are an inevitable consequence of interstellar turbulence but could also be generated by variability and anisotropy in a precursor wind from the GRB progenitor. Detection of their properties could provide important clues about the environments of GRB sources. We apply our calculations to GRB 990510, where an rms scatter of $\sim 2 \%$ was observed for the optical flux fluctuations on the $0.1-2 \mathrm{hr}$ timescale during the first day of the afterglow, consistent with it being due entirely to photometric noise. The resulting upper limits on the density fluctuations on scales of $\sim 20-200$ AU around the source of GRB 990510 are lower than the inferred fluctuation amplitude on similar scales in the Galactic interstellar medium. Hourly monitoring of future optical afterglows might therefore reveal fractional flux fluctuations at the level of a few percent.
\end{abstract}

Subject headings: gamma rays: bursts - turbulence

\section{INTRODUCTION}

Almost all well-localized gamma-ray burst (GRB) sources have shown afterglow emission in X-rays for several hours (see Piran 1999 for a review). Often the emission persists on longer timescales at lower photon energies, peaking in the optical on a timescale of days and in the radio on a timescale of weeks or longer. This long-lasting afterglow emission is most naturally explained as synchrotron emission from a relativistic blast wave, produced by the GRB explosion in an external medium (see, e.g., Paczyński \& Rhoads 1993; Katz 1994; Mészáros \& Rees 1993, 1997; Waxman 1997a, 1997b). The emission frequency declines with time because of the deceleration of the shock wave (Blandford \& McKee 1976) and the corresponding reduction in the characteristic electron energy and magnetic field amplitude behind the shock. The external medium could be either the interstellar medium (ISM) of the host galaxy (Waxman 1997a) or a precursor wind from the GRB progenitor (Chevalier \& Li 2000).

Previous theoretical models of the afterglow emission have assumed for simplicity that the density profile of the external medium is smooth, i.e., uniform in the case of the ISM or power law with radius in the case of a progenitor wind. However, this simplifying assumption is not expected to hold in realistic situations. The ISM is known to exhibit inhomogeneities because of turbulence, and stellar winds may vary in time and in solid angle. Since the emitted afterglow flux depends on the instantaneous number of shocked electrons, any density inhomogeneities are expected to induce temporal fluctuations in the afterglow light curve. Observations of these fluctuations could provide additional constraints on the nature of the surrounding medium and the GRB progenitor.

In this paper we derive the relation between the spatial power spectrum of density fluctuations in the ambient medium surrounding the GRB source and the Fourier transform of temporal fluctuations in the afterglow flux. We focus on smallamplitude (linear) inhomogeneities, as those provide the minimum source of afterglow flux fluctuations. Section 2 presents the formalism used in our derivation, and $\S 3$ describes our numerical results. We summarize our main conclusions in $\S 4$. For simplicity, we consider the case where the unperturbed ambient medium is uniform, as for a background ISM. The particle density inferred from detailed modeling of some GRB afterglows is in the range $\sim 0.1-1 \mathrm{~cm}^{-3}$ (e.g., Wijers \& Galama 1999; Waxman 1997a, 1997b), as expected for the ISM of their host galaxies.

\section{FORMALISM}

The relativistic fireball produced by a GRB explosion starts to decelerate at the radius where the energy of the ambient gas swept by the fireball is comparable to its energy output (e.g., Waxman 1997a, 1997b),

$$
r_{c}=2 \times 10^{16}\left(\frac{E_{52} t_{10}}{n_{1}}\right)^{1 / 4} \mathrm{~cm},
$$

where $E_{52}$ is the hydrodynamic energy release in units of $10^{52} \mathrm{ergs}, t_{10}$ is the duration of the GRB in units of $10 \mathrm{~s}$, and $n_{1}$ is the proton density of the ambient medium in units of $1 \mathrm{~cm}^{-3}$. At larger radii, the shock follows the adiabatic self-similar evolution (Blandford \& McKee 1976) whereby its Lorentz factor declines with radius as

$$
\Gamma=\Gamma_{c}\left(\frac{r}{r_{c}}\right)^{-3 / 2}
$$


and

$$
\Gamma_{c}=270\left(\frac{E_{52}}{t_{10}^{3} n_{1}}\right)^{1 / 8} .
$$

The global spectral characteristics of GRB afterglows are naturally explained in terms of synchrotron emission by shockaccelerated electrons from this decelerating relativistic shock (see, e.g., Wijers, Rees, \& Mészáros 1997; Waxman 1997a, 1997b; Sari, Piran, \& Narayan 1998).

Figure 1 illustrates the geometry of the emission from an infinitesimal volume element in spherical coordinates $d V=r^{2} d r d \mu d \phi$ at a distance $D$ from the observer. Here $\mu=\cos \theta$ and $\theta=0$ along the line of sight. We define the emission coefficient $j_{v^{\prime}}^{\prime}$ to be the power emitted per unit frequency per unit volume per steradian in the rest frame of the outflowing material. We use prime to denote quantities in the local rest frame of the emitting material, while unprimed quantities are measured in the rest frame of the ISM. Note that $j_{v} / v^{2}$ is Lorentz invariant (Rybicki \& Lightman 1979, p. 147). For a spherically symmetric expansion of material that emits isotropically in its local rest frame, we have $v^{\prime}=v \gamma(1-\beta \mu)$ and $j_{v^{\prime}}^{\prime}=P^{\prime}\left(v^{\prime}, r, t\right) / 4 \pi$, where $\gamma$ and $\beta c$ are the Lorentz factor and the velocity of the emitting matter, respectively. A photon emitted at time $t$ and place $r$ in the ISM frame will reach the detector at a time $T$ given by

$$
T_{z}=\frac{T}{1+z}=t-\frac{r \mu}{c},
$$

where $z$ is the cosmological redshift of the GRB and $T$ is chosen such that a photon emitted at the origin at $t=0$ will arrive at the detector at $T=0$. Thus, we have (Granot, Piran, \& Sari 1999)

$$
F(v, T)=\frac{1+z}{4 \pi d_{L}^{2}} \int_{0}^{2 \pi} d \phi \int_{-1}^{1} d \mu \int_{0}^{\infty} r^{2} d r \frac{P^{\prime}\left[v \gamma(1-\beta \mu), r, T_{z}+r \mu / c\right]}{\gamma^{2}(1-\beta \mu)^{2}},
$$

where $d_{L}$ is the luminosity distance to the GRB and $\gamma, \beta$, and $\mu$ are evaluated at the time $t$ implied by equation (4).

Most of the shocked material is concentrated in a thin shell behind the shock front. The characteristic thickness of the shell is $\Delta \sim R / 10 \gamma^{2}$ in the ISM frame, where $\gamma=\Gamma / \sqrt{2}$ is the Lorentz factor of the material just behind the shock. In the following, we will assume that the observed radiation originates from the thin shell of thickness $\Delta=\eta R / \gamma^{2}$ behind the shock, inside of which the Lorentz factor, the particle density, and the energy density of shocked ISM obtain the following values:

$$
n^{\prime}=4 \gamma n, \quad e^{\prime}=4 \gamma^{2} n m_{p} c^{2},
$$

where $n$ is the number density of the unshocked ambient ISM in its local rest frame and $m_{p}$ is the proton mass. The actual value of $\eta$ depends not only on the hydrodynamics but also on the behavior of the magnetic field and the shock-accelerated electrons that determine the local emissivity.

The volume integration expressed in equation (5) should be taken over the region occupied by the emitting shell at a given observed time, as illustrated in Figure 2. Because of relativistic beaming, the observed radiation originates from a small angle along the line of sight, $\theta<1 / \gamma$. Hence, we can set the upper limit in the integration over $\theta$ to be $2 / \gamma_{b}$, where $\gamma_{b}$ is the Lorentz factor of the shell at point $b$ on the plot. For a given observed time $T$, the outer boundary $a b c$ of the integration region is defined by the relation (Granot et al. 1999)

$$
R_{\text {outer }}=\frac{c T_{z}}{1-\mu+1 /\left(16 \gamma^{2}\right)},
$$

where $\gamma=\gamma\left(\boldsymbol{R}_{\text {outer }}\right)$. Photons originating at this boundary are emitted from the front of the shell and arrive at the detector at the same time $T$ (although they are emitted at different times). Similarly, the inner boundary of the integration region is described by

$$
R_{\text {inner }}=\frac{c T_{z}}{1-\mu+(\eta+1 / 16) / \gamma^{2}},
$$

with $\gamma=\gamma\left(\boldsymbol{R}_{\text {inner }}\right)$, and where the associated photons are emitted from the back of the shell. Note that, although the emitting shell is thin, the region contributing to the observed flux at a given time is rather extended; this has important consequences with respect to the spatial scale over which density fluctuations could affect the observed flux. Furthermore, consider a point

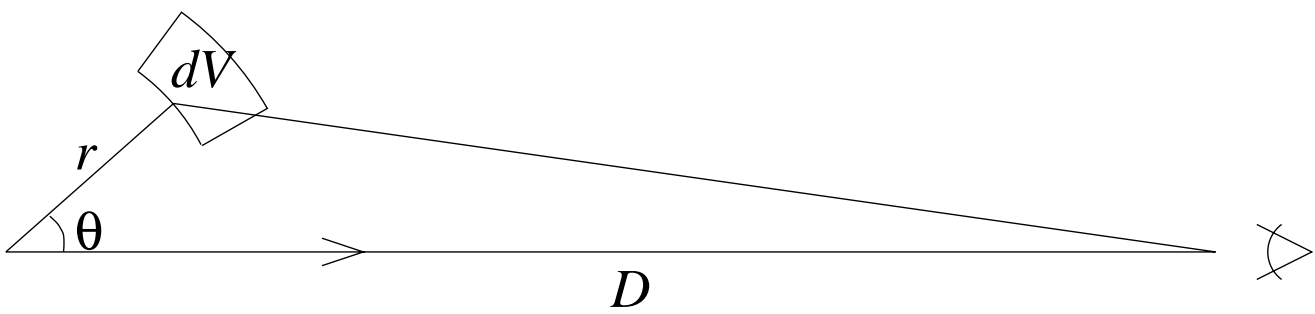

FIG. 1.-Coordinate system for the integration of the emitted afterglow flux 


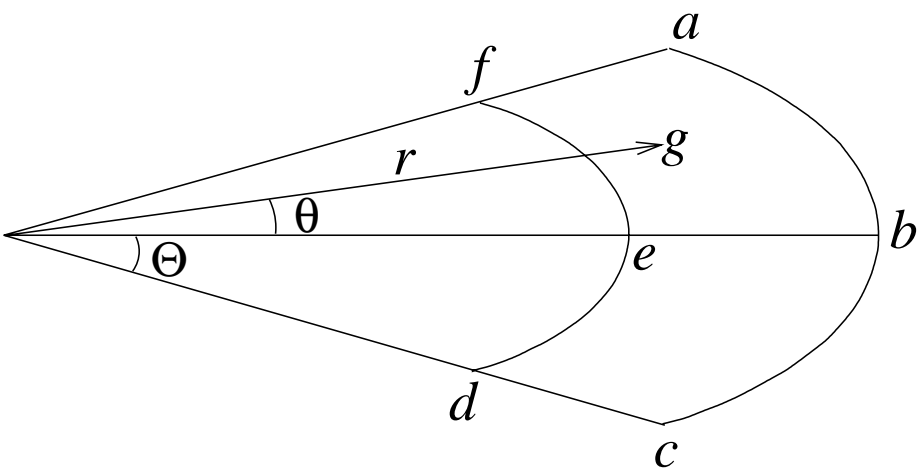

FIG. 2.-Notations and geometry of the integration region

$g=(r, \theta)$ inside the integration region. A photon emitted from this point at time $t=T_{z}+r \mu / c$ will arrive at the detector at time $T$. Since the emitting shell is very thin, the point $g$ is very close to the shock front at time $t$. Thus, the radius of the shock at time $t$ is approximately equal to $r$. This fact will be used in our calculation.

Next, we derive the local emissivity due to synchrotron radiation. We assume that the energy densities of the shockaccelerated electrons and the magnetic field are fixed fractions of the internal energy density behind the shock front, $e_{e}^{\prime}=\epsilon_{e} e^{\prime}$ and $e_{B}^{\prime}=\epsilon_{B} e^{\prime}$, and that the shock produces a power-law distribution of accelerated electrons with a number density per Lorentz factor of $N\left(\gamma_{e}\right)=K \gamma_{e}^{-p}$ for $\gamma_{e} \geq \gamma_{\min }$, where

$$
\gamma_{\min }=\left(\frac{p-2}{p-1}\right) \frac{\epsilon_{e} e^{\prime}}{n^{\prime} m_{e} c^{2}}, \quad K=(p-1) n^{\prime} \gamma_{\min }^{p-1} .
$$

Then the emissivity can be approximated as

$$
P^{\prime}=H_{1} \gamma \beta^{2} n^{4 / 3}\left(v^{\prime}\right)^{1 / 3}, \quad v^{\prime}<v_{\min }^{\prime},
$$

and

$$
P^{\prime}=H_{2} \gamma^{(3 p+1) / 2} \beta^{2} n^{(p+5) / 4}\left(v^{\prime}\right)^{-(p-1) / 2}, \quad v^{\prime}>v_{\min }^{\prime},
$$

where $v_{\min }^{\prime}=v_{\mathrm{syn}}^{\prime}\left(\gamma_{\min }\right)$ is the synchrotron frequency of an electron with the minimal Lorentz factor, and

$$
H_{1}=32.4 \times \frac{(p-1)^{5 / 3}}{(3 p-1)(p-2)^{2 / 3}} \sigma_{T} \frac{\epsilon_{B}^{1 / 3}}{\epsilon_{e}^{2 / 3}} \frac{m_{e}^{2} c^{3}}{m_{p}^{1 / 3} q_{e}^{4 / 3}}
$$

and

$$
H_{2}=39.9 \times 1.9^{(p-1) / 2} \times \frac{(p-2)^{p-1}}{(3 p-1)(p-1)^{p-2}} \sigma_{T} \epsilon_{B}^{(p+1) / 4} \epsilon_{e}^{p-1} \frac{m_{p}^{(5 p-3) / 4} c^{3}}{m_{e}^{(3 p-5) / 2} q_{e}^{(3-p) / 2}}
$$

are constants. In addition, note that the frequency at which the observed flux peaks is $v_{\text {peak }} \sim v_{\min }$.

Substituting equations (10) and (11) into equation (5), respectively, and making use of the fact that for a highly relativistic system, $\theta \leq 2 / \gamma_{b} \ll 1$, and so

$$
d \mu \approx-\theta d \theta, \quad 1-\beta \mu \approx \frac{1}{2 \gamma^{2}}+\frac{\theta^{2}}{2},
$$

we get the afterglow flux at a frequency $v$,

$$
F(v, T)=\frac{(1+z) H_{1} v^{1 / 3}}{4 \pi d_{L}^{2}} \int_{0}^{2 \pi} d \phi \int_{0}^{2 / \gamma b} d \theta \int_{R_{\text {inner }}}^{R_{\text {outer }}} d r \frac{n^{4 / 3} \theta r^{2}}{\gamma^{2 / 3}\left(1 / 2 \gamma^{2}+\theta^{2} / 2\right)^{5 / 3}}, \quad v<v_{\text {peak }},
$$

and

$$
F(v, T)=\frac{(1+z) H_{2} v^{-(p-1) / 2}}{4 \pi d_{L}^{2}} \int_{0}^{2 \pi} d \phi \int_{0}^{2 / \gamma b} d \theta \int_{R_{\text {inner }}}^{R_{\text {outer }}} d r \frac{n^{(p+5) / 4} \gamma^{p-1} \theta r^{2}}{\left(1 / 2 \gamma^{2}+\theta^{2} / 2\right)^{(p+3) / 2}}, \quad v>v_{\text {peak }} .
$$

All emission frequencies under consideration are assumed to be below the cooling frequency, $v_{c}$ (i.e., not affected by the rapid cooling of the high-energy tail of the electron distribution), and also well above the synchrotron self-absorption frequency, $v_{a}$ (see Sari et al. 1998 for more details).

At point $b$ in Figure 2, the radius of the shock front is $R_{b}$, the Lorentz factor of the shell behind the shock is $\gamma_{b}$, and the time $T$ when photons emitted at point $b$ reach the observer is related to these quantities by

$$
\frac{T}{(1+z)}=\frac{R_{b}}{16 \gamma_{b}^{2} c} \text {. }
$$


Note that this time is different from the observation time, $T_{\text {obs }}$, which is defined as the arrival time of most photons emitted from the shell of radius $R_{b}$. This is because most photons are emitted from a cone of opening angle $\sim 1 / \gamma_{b}$ around the line of sight and they suffer a longer time delay $\sim R_{b} / 2 \gamma_{b}^{2} c$ than the photons emitted on the line of sight (Waxman 1997c). The observation time is

$$
\frac{T_{\mathrm{obs}}}{(1+z)} \approx \frac{R_{b}}{2 \gamma_{b}^{2} c}=8 \frac{T}{(1+z)}
$$

We can use $R_{b}$ to normalize equations (15) and (16). Based on the scaling, $\gamma \propto r^{-3 / 2}$, we get

$$
\frac{\gamma}{\gamma_{b}}=\left(\frac{r}{R_{b}}\right)^{-3 / 2}
$$

Using equations (17) and (19), we can rewrite equations (7) and (8) as follows:

$$
X_{\text {outer }}^{4}+8 \gamma_{b}^{2} \theta^{2} X_{\text {outer }}-1=0
$$

and

$$
(16 \eta+1) X_{\text {inner }}^{4}+8 \gamma_{b}^{2} \theta^{2} X_{\text {inner }}-1=0,
$$

where $x=r / R_{b}, X_{\text {outer }} \equiv R_{\text {outer }} / R_{b}$, and $X_{\text {inner }} \equiv R_{\text {inner }} / R_{b}$. In these notations, equations (15) and (16) obtain the form

$$
F(v, T)=\frac{(1+z) H_{1} v^{1 / 3} R_{b}^{3}}{4 \pi d_{L}^{2} \gamma_{b}^{2 / 3}} \int_{0}^{2 \pi} d \phi \int_{0}^{2 / \gamma_{b}} d \theta \int_{X_{\text {inner }}\left(\theta, \gamma_{b}\right)}^{X_{\text {outer }}\left(\theta, \gamma_{b}\right)} \frac{n^{4 / 3} \theta x^{3} d x}{\left(x^{3} / 2 \gamma_{b}^{2}+\theta^{2} / 2\right)^{5 / 3}}, \quad v<v_{\text {peak }},
$$

and

$$
F(v, T)=\frac{(1+z) H_{2} v^{-(p-1) / 2} R_{b}^{3} \gamma_{b}^{p-1}}{4 \pi d_{L}^{2}} \int_{0}^{2 \pi} d \phi \int_{0}^{2 / \gamma_{b}} d \theta \int_{X_{\text {inner }}\left(\theta, \gamma_{b}\right)}^{X_{\text {outer }}\left(\theta, \gamma_{b}\right)} \frac{n^{(p+5) / 4} \theta d x}{x^{(3 p-7) / 2}\left(x^{3} / 2 \gamma_{b}^{2}+\theta^{2} / 2\right)^{(p+3) / 2}}, \quad v>v_{\text {peak }},
$$

where $X_{\text {inner }}\left(\theta, \gamma_{b}\right)$ and $X_{\text {outer }}\left(\theta, \gamma_{b}\right)$ can be obtained by solving equations (20) and (21), and $R_{b}$ and $\gamma_{b}$ are functions of the observed time $T$. At a later time $\tilde{T}=T+\tau$, the shock front moves from $b$ to $\tilde{b}$, while the Lorentz factor and radius of the shell change to $\tilde{\gamma}_{b}$ and $\tilde{R}_{b}$, respectively, with

$$
\frac{R_{b}}{\widetilde{R}_{b}}=\left(\frac{T}{\widetilde{T}}\right)^{1 / 4}, \quad \frac{\gamma_{b}}{\tilde{\gamma}_{b}}=\left(\frac{T}{\widetilde{T}}\right)^{-3 / 8} .
$$

Equations (22) and (23) can be written in the generalized form

$$
F(T)=\int G(\boldsymbol{r}) n^{y}(\boldsymbol{r}) d \boldsymbol{r},
$$

where the density $n(\boldsymbol{r})$ may fluctuate and

$$
y=\left\{\begin{array}{cl}
4 / 3 & \text { for } v<v_{\text {peak }} \\
(p+5) / 4 & \text { for } v>v_{\text {peak }}
\end{array}\right.
$$

Thus, we get

$$
\langle F(T) F(T+\tau)\rangle=\left\langle\int G(\boldsymbol{r}) n^{y}(\boldsymbol{r}) d \boldsymbol{r} \int G(\tilde{\boldsymbol{r}}) n^{y}(\tilde{\boldsymbol{r}}) d \tilde{\boldsymbol{r}}\right\rangle=\int d \boldsymbol{r} \int d \tilde{\boldsymbol{r}} G(\boldsymbol{r}) G(\tilde{\boldsymbol{r}})\left\langle n^{y}(\boldsymbol{r}) n^{y}(\tilde{\boldsymbol{r}})\right\rangle .
$$

The angular brackets in the above equation reflect an average over an ensemble of afterglows with identical source properties, exploding at different places in the ISM. The ergodic assumption implies that an average over many such systems would be equivalent to an average over time for the explosion if the ISM is in a stationary statistical state (Reif 1965, pp. 583-585). For the ensemble average of the right-hand side, we have

$$
\begin{aligned}
\left\langle n^{y}(\boldsymbol{r}) n^{y}(\tilde{\boldsymbol{r}})\right\rangle & =\langle n\rangle^{2 y}\left\langle[1+\delta(\boldsymbol{r})]^{y}[1+\delta(\tilde{\boldsymbol{r}})]^{y}\right\rangle \\
& \approx\langle n\rangle^{2 y}\left\langle 1+\frac{y(y-1)}{2} \delta^{2}(\boldsymbol{r})+\frac{y(y-1)}{2} \delta^{2}(\tilde{\boldsymbol{r}})+y^{2} \delta(\boldsymbol{r}) \delta(\tilde{\boldsymbol{r}})\right\rangle \\
& =\langle n\rangle^{2 y}\left[1+y(y-1) \xi_{0}+y^{2} \xi(\boldsymbol{r}-\tilde{\boldsymbol{r}})\right],
\end{aligned}
$$

where

$$
\delta(\boldsymbol{r})=\frac{n(\boldsymbol{r})-\langle n\rangle}{\langle n\rangle},
$$

$\xi(\boldsymbol{r}-\tilde{\boldsymbol{r}}) \equiv\langle\delta(\boldsymbol{r}) \delta(\tilde{\boldsymbol{r}})\rangle$ is the ensemble-averaged autocorrelation function of the density fluctuations, and $\xi_{0}=\left\langle\delta^{2}(\boldsymbol{r})\right\rangle=$ $\left\langle\delta^{2}(\tilde{r})\right\rangle$. As mentioned before, we consider only small (linear) density fluctuations. In equation (28), we have implicitly assumed $\delta(\boldsymbol{r}) \ll 1$. 
Similarly we have

$$
\langle F(T)\rangle\langle F(T+\tau)\rangle=\int d \boldsymbol{r} \int d \tilde{\boldsymbol{r}} G(\boldsymbol{r}) G(\tilde{\boldsymbol{r}})\left\langle n^{y}(\boldsymbol{r})\right\rangle\left\langle n^{y}(\tilde{\boldsymbol{r}})\right\rangle,
$$

and

$$
\left\langle n^{y}(\boldsymbol{r})\right\rangle\left\langle n^{y}(\tilde{\boldsymbol{r}})\right\rangle \approx\langle n\rangle^{2 y}\left[1+y(y-1) \xi_{0}\right] .
$$

The statistical properties of the ambient gas inhomogeneities in the vicinity of GRB sources are highly uncertain, so we adopt the minimal number of free parameters to describe the autocorrelation function, namely, we write

$$
\xi(r)=\xi_{0} \exp \left(-\frac{r}{r_{0}}\right),
$$

where $r_{0}$ is the scale length of the density autocorrelation function. For simplicity, we ignore deviations of the expanding shell from spherical symmetry. Our calculation focuses on scales much smaller than the size of the emission region (see $\S 3$ ), so the cumulative effect of many small-scale patches of density perturbations averages out during the expansion history of the shell. At different points inside the shell, the particle density and energy density (described by eq. [6]) might be temporarily above or below their average values, but the total energy is conserved.

We can now define the autocorrelation function of the temporal fluctuations in the afterglow flux as

$$
\zeta(\tau)=\langle\Delta(T) \Delta(T+\tau)\rangle=\frac{\langle F(T) F(T+\tau)\rangle}{\langle F(T)\rangle\langle F(T+\tau)\rangle}-1,
$$

where we consider fluctuations on timescales much shorter than the evolution time of the afterglow light curve, $\tau \ll T$, and where

$$
\Delta(T)=\frac{F(T)-\langle F(T)\rangle}{\langle F(T)\rangle}
$$

We now define

$$
g\left(x, \theta, \gamma_{b}\right)= \begin{cases}\frac{\theta x^{3}}{\left(x^{3} / 2 \gamma_{b}^{2}+\theta^{2} / 2\right)^{5 / 3}} & \text { for } v<v_{\text {peak }}, \\ \frac{\theta}{x^{(3 p-7) / 2}\left(x^{3} / 2 \gamma_{b}^{2}+\theta^{2} / 2\right)^{(p+3) / 2}} & \text { for } v>v_{\text {peak }} .\end{cases}
$$

One of the integrals of interest is

$$
I_{1}=\int_{0}^{2 \pi} d \phi \int_{0}^{2 / \gamma_{b}} d \theta \int_{X_{\text {inner }}\left(\theta, \gamma_{b}\right)}^{X_{\text {outer }}\left(\theta, \gamma_{b}\right)} g\left(x, \theta, \gamma_{b}\right) d x \int_{0}^{2 \pi} d \tilde{\phi} \int_{0}^{2 / \tilde{\gamma}_{b}} d \tilde{\theta} \int_{\tilde{X}_{\text {inner }}\left(\tilde{\theta}, \tilde{\gamma}_{b}\right)}^{\tilde{X}_{\text {outer }}\left(\tilde{\theta}, \tilde{\gamma}_{b}\right)} g\left(\tilde{x}, \tilde{\theta}, \tilde{\gamma}_{b}\right) d \tilde{x},
$$

where $\tilde{X}_{\text {inner }}\left(\tilde{\theta}, \tilde{\gamma}_{b}\right)$ and $\tilde{X}_{\text {outer }}\left(\tilde{\theta}, \tilde{\gamma}_{b}\right)$ are found by solving equations (20) and (21). A second relevant integral is

$$
I_{2}=\int_{0}^{2 \pi} d \phi \int_{0}^{2 / \gamma_{b}} d \theta \int_{X_{\text {inner }}\left(\theta, \gamma_{b}\right)}^{X_{\text {outer }}\left(\theta, \gamma_{b}\right)} g\left(x, \theta, \gamma_{b}\right) d x \int_{0}^{2 \pi} d \tilde{\phi} \int_{0}^{2 / \tilde{\gamma}_{b}} d \tilde{\theta} \int_{\tilde{X}_{\text {inner }}\left(\tilde{\theta}, \tilde{X}_{b}\right)}^{\tilde{X}_{\text {outer }}\left(\tilde{\theta}, \tilde{\gamma}_{b}\right)} y^{2} \xi(|\boldsymbol{r}-\tilde{\boldsymbol{r}}|) g\left(\tilde{x}, \tilde{\theta}, \tilde{\gamma}_{b}\right) d \tilde{x},
$$

where

$$
|\boldsymbol{r}-\tilde{\boldsymbol{r}}|=\left[\left(x \boldsymbol{R}_{b} \sin \theta-\tilde{x} \tilde{\boldsymbol{R}}_{b} \sin \tilde{\theta} \cos \tilde{\phi}\right)^{2}+\left(\tilde{x} \tilde{\boldsymbol{R}}_{b} \sin \tilde{\theta} \sin \tilde{\phi}\right)^{2}+\left(x \boldsymbol{R}_{b} \cos \theta-\tilde{x} \tilde{\boldsymbol{R}}_{b} \cos \tilde{\theta}\right)^{2}\right]^{1 / 2} .
$$

Using the above integrals we may write

$$
\zeta(\tau)=\langle\Delta(T) \Delta(T+\tau)\rangle=I_{2} / I_{1}
$$

\section{NUMERICAL RESULTS}

We evaluated numerically the integrals in equations (36) and (37) using a Gaussian quadrature method. For each of the two different frequency regions $\left(v<v_{\text {peak }}\right.$ and $\left.v>v_{\text {peak }}\right)$, we considered six different cases with values of $\gamma_{b}$ of 100, 22, and 3, which correspond to peak afterglow emission in the $X$-ray, optical, and radio wavelength regimes, and for each $\gamma_{b}$, we considered two different cases, $r_{0} / R_{b}=10^{-3}$ and $r_{0} / R_{b}=10^{-2}$. The values of $\gamma_{b}, R_{b}$, and $v_{\text {peak }}$, the frequency at which the observed flux peaks at a given observation time $T_{\text {obs }}$, are given by (Granot et al. 1999),

$$
\gamma_{b}=7.96\left(\frac{E_{52}}{n_{1}}\right)^{1 / 8}\left(\frac{T_{\text {obs,days }}}{1+z}\right)^{-3 / 8},
$$


TABLE 1

VALUES OF $T_{\text {obs }}, R_{b}$, AND $v_{\text {peak }}$ FOR THE THREE CHOICES OF $\gamma_{b}$

\begin{tabular}{cccc}
\hline \hline$\gamma_{b}$ & $T_{\text {obs }} /\left\{E_{52}^{1 / 3} n_{1}^{-1 / 3}[(1+z) / 2]\right\}$ & $\begin{array}{c}R_{b} /\left(E_{52}^{1 / 3} n_{1}^{-1 / 3}\right) \\
(\mathrm{cm})\end{array}$ & $\begin{array}{c}v_{\text {peak }} /\left\{n_{1}^{1 / 2}[(1+z) / 2]^{-1}\right\} \\
(\mathrm{Hz})\end{array}$ \\
\hline $100 \ldots \ldots$ & $202.6 \mathrm{~s}$ & $6.1 \times 10^{16}$ & $2.9 \times 10^{17}$ \\
$22 \ldots \ldots \ldots$ & $3.2 \mathrm{hr}$ & $1.7 \times 10^{17}$ & $6.7 \times 10^{14}$ \\
$3 \ldots \ldots \ldots$ & $27.0 \mathrm{days}$ & $6.3 \times 10^{17}$ & $2.3 \times 10^{11}$ \\
\hline
\end{tabular}

NoTE.-The values of $E_{52}, n_{1}$, and $z$ are left as free parameters.

$$
R_{b}=3.29 \times 10^{17}\left[\frac{E_{52} T_{\text {obs,days }}}{n_{1}(1+z)}\right]^{1 / 4} \mathrm{~cm}
$$

and

$$
v_{\text {peak }}=7.29 \times 10^{15} \sqrt{1+z} \frac{\phi_{\text {peak }}(p)}{\phi_{\text {peak }}(2.5)} \frac{f(p)}{f(2.5)} \epsilon_{B}^{1 / 2} \epsilon_{e}^{2} E_{52}^{1 / 2} T_{\text {obs,days }}^{-3 / 2} \mathrm{~Hz},
$$

where $\phi_{\text {peak }}$ is a slowly decreasing function of $p, f(p) \equiv[(p-2) /(p-1)]^{2}$, and $T_{\text {obs, days }}$ is the observation time in days and is related to $T$ by equation (18).

In our numerical calculations we assume $p=2.5$ (Granot et al. 1999; Sari, Piran, \& Halpern 1999), $\epsilon_{B}=0.1, \epsilon_{e}=0.1$, and $\eta=0.1$. Table 1 shows the associated $T, T_{\mathrm{obs}}, R_{b}$, and $v_{\text {peak }}$ for the three choices of $\gamma_{b}$, with $E_{52}, n_{1}$, and $z$ as free parameters.

Figures 3 and 4 show the numerical results for the six cases mentioned above, with Figure 3 corresponding to $v<v_{\text {peak }}$ and Figure 4 corresponding to $v>v_{\text {peak }}$. We plot the square root of the autocorrelation function for the temporal fluctuations of the afterglow flux $\zeta\left(\tau_{\text {obs }}\right)$, as normalized by the unknown amplitude of the fractional density fluctuations in the ambient medium, $\xi_{0}^{1 / 2}$. We normalize $r_{0}$ by $R_{b}$, which is different for the three different cases of $\gamma_{b}$.

The value of $\left[\zeta\left(\tau_{\mathrm{obs}}\right)\right]^{1 / 2}$ at $\tau_{\mathrm{obs}}=0$ provides the typical amplitude of the fluctuations in the observed flux. The characteristic period of the fluctuations is $\tau_{1 / 2, \mathrm{obs}}$, the time over which $\zeta\left(\tau_{\mathrm{obs}}\right)$ drops to one-half its maximum value. We list the derived values
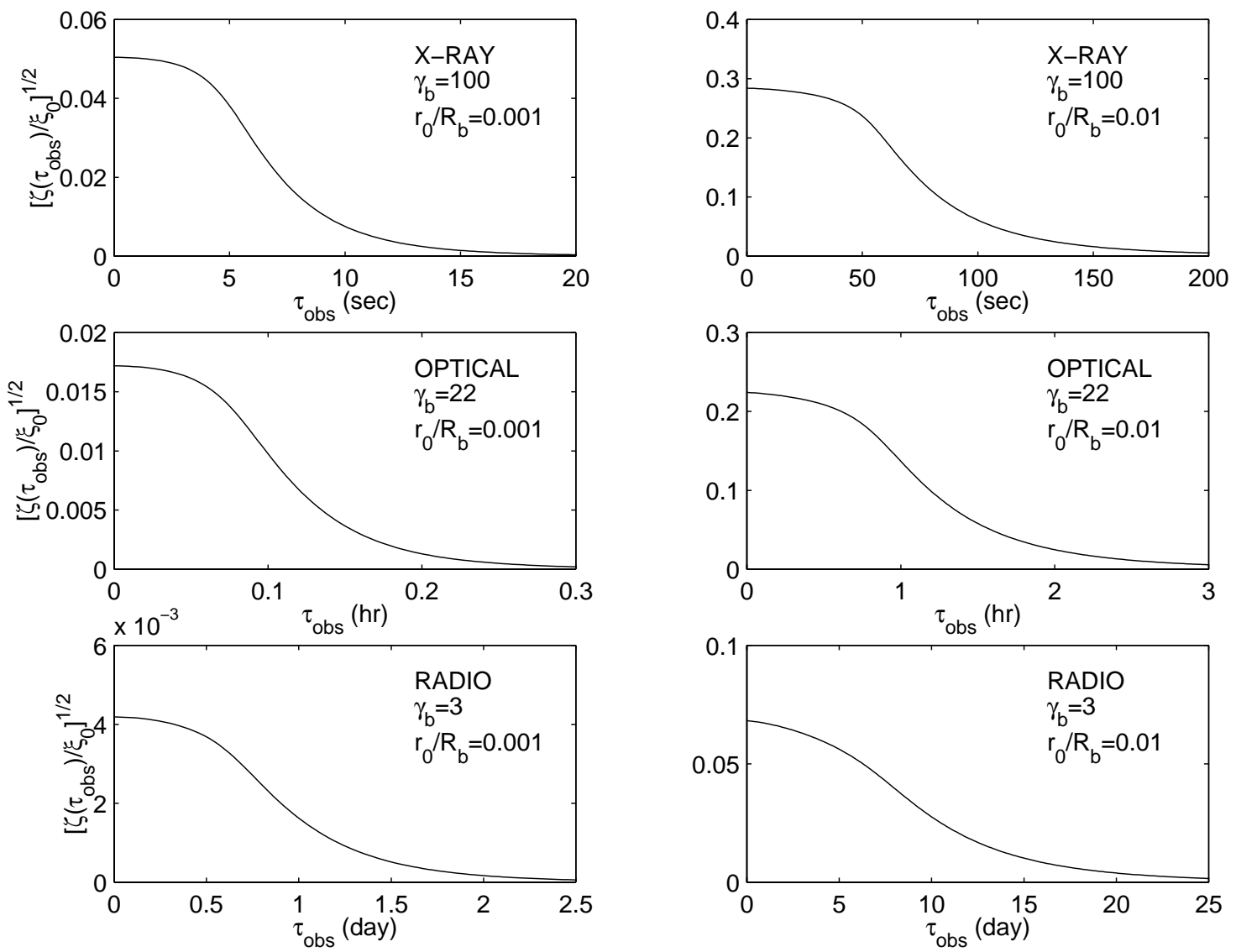

FIG. 3.-Temporal correlation function for variations of the afterglow flux $\left[\zeta\left(\tau_{\mathrm{obs}}\right) / \xi_{0}\right]^{1 / 2}$ as a function of $\tau_{\mathrm{obs}}$ for $v<v_{\text {peak }}$, where $\tau_{\mathrm{obs}}$ scales as $E_{52}^{1 / 3} n_{1}^{-1 / 3}[(1+z) / 2]$. The six panels correspond to six cases for different values of parameters, as shown next to each panel. 

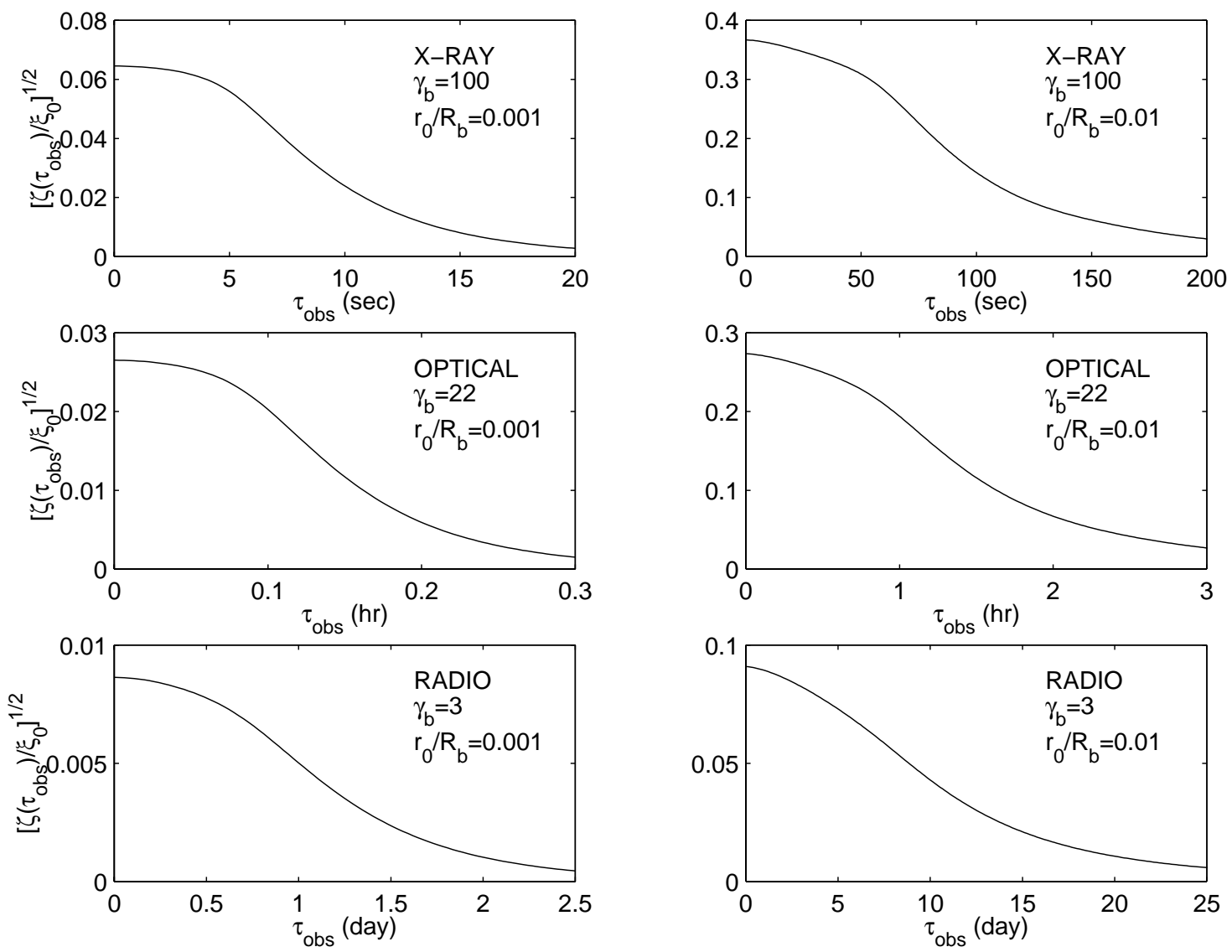

FIG. 4. - Same as Fig. 3 but for $v>v_{\text {peak }}$

of these parameters in Tables 2 and 3. The tables imply that (1) for a given $r_{0} / R_{b},\left[\zeta(0) / \xi_{0}\right]^{1 / 2}$ decreases with decreasing $\gamma_{b}$ and (2) for a given $\gamma_{b},\left[\zeta(0) / \xi_{0}\right]^{1 / 2}$ decreases with decreasing $r_{0} / R_{b}$. Poisson statistics implies that density fluctuations with an amplitude $\delta_{0} \ll 1$ on a correlation length $l$ will induce an average fluctuation amplitude $\sim \delta_{0} / \sqrt{N}$ in a region of size $L \gg l$, where $N \sim(L / l)^{3}$ is the number of independent regions of positive or negative density fluctuations in the sampled volume. This explains the qualitative trend of $[\zeta(0)]^{1 / 2}$ to decrease as the value of $r_{0}$ is lowered. However, $[\zeta(0)]^{1 / 2}$ is not proportional

TABLE 2

VALUES OF $\left[\zeta(0) / \xi_{0}\right]^{1 / 2}, \tau_{1 / 2, \text { obs }} / T_{\text {obs }}$, AND $\tau_{1 / 2, \text { obs }}$ FOR SIX CASES IN THE $v<v_{\text {peak }}$ REGION

\begin{tabular}{|c|c|c|c|c|c|c|}
\hline Case & $\gamma_{b}$ & $r_{0} / R_{b}$ & $\begin{array}{c}r_{0} /\left(E_{52}^{1 / 3} n_{1}^{-1 / 3}\right) \\
(\mathrm{cm})\end{array}$ & {$\left[\zeta(0) / \xi_{0}\right]^{1 / 2}$} & $\tau_{1 / 2, \mathrm{obs}} / T_{\mathrm{obs}}$ & $\tau_{1 / 2, \mathrm{obs}} /\left\{E_{52}^{1 / 3} n_{1}^{-1 / 3}[(1+z) / 2]\right\}$ \\
\hline $1 \ldots \ldots$ & 100 & 0.001 & $6.1 \times 10^{13}$ & 0.05 & 0.026 & $5.3 \mathrm{~s}$ \\
\hline $2 \ldots \ldots$ & 100 & 0.01 & $6.1 \times 10^{14}$ & 0.28 & 0.30 & $61.6 \mathrm{~s}$ \\
\hline $3 \ldots \ldots$ & 22 & 0.001 & $1.7 \times 10^{14}$ & 0.017 & 0.027 & $0.086 \mathrm{hr}$ \\
\hline $4 \ldots \ldots$ & 22 & 0.01 & $1.7 \times 10^{15}$ & 0.22 & 0.30 & $0.94 \mathrm{hr}$ \\
\hline $5 \ldots \ldots$ & 3 & 0.001 & $6.3 \times 10^{14}$ & 0.004 & 0.026 & 0.70 days \\
\hline $6 \ldots \ldots$ & 3 & 0.01 & $6.3 \times 10^{15}$ & 0.07 & 0.26 & 7.0 days \\
\hline
\end{tabular}

NoTE.-The values of $E_{52}, n_{1}$, and $z$ are left as free parameters.

TABLE 3

VALUES OF $\left[\zeta(0) / \xi_{0}\right]^{1 / 2}, \tau_{1 / 2, \text { obs }} / T_{\text {obs }}$, AND $\tau_{1 / 2, \text { obs }}$ FOR SIX CASES IN THE $v>v_{\text {peak }}$ REGION

\begin{tabular}{lrlccc}
\hline \hline Case & $\gamma_{b}$ & $r_{0} / R_{b}$ & {$\left[\zeta(0) / \xi_{0}\right]^{1 / 2}$} & $\tau_{1 / 2, \text { obs }} / T_{\text {obs }}$ & $\tau_{1 / 2, \text { obs }} /\left\{E_{52}^{1 / 3} n_{1}^{-1 / 3}[(1+z) / 2]\right\}$ \\
\hline $1 \ldots \ldots$ & 100 & 0.001 & 0.06 & 0.033 & $6.7 \mathrm{~s}$ \\
$2 \ldots \ldots$ & 100 & 0.01 & 0.37 & 0.33 & $67 \mathrm{~s}$ \\
$3 \ldots \ldots$ & 22 & 0.001 & 0.026 & 0.034 & $0.11 \mathrm{hr}$ \\
$4 \ldots \ldots$ & 22 & 0.01 & 0.27 & 0.31 & $1.0 \mathrm{hr}$ \\
$5 \ldots \ldots$ & 3 & 0.001 & 0.009 & 0.031 & 0.84 days \\
$6 \ldots \ldots$ & 3 & 0.01 & 0.09 & 0.24 & 6.5 days \\
\hline
\end{tabular}

NoTE.-The values of $E_{52}, n_{1}$ and $z$ are left as free parameters. 
to $r_{0}^{3 / 2}$ in our problem because the different uncorrelated regions within the integration volume have different weights in their contribution to the total flux.

\section{CONSTRAINTS FROM GRB 990510}

Stanek et al. (1999) have monitored the optical afterglow of GRB 990510 on a subhour basis and obtained an rms scatter of 0.02 mag for the $B V R I$ observations during its first day, consistent with the scatter being due entirely to photometric noise (see also Hjorth et al. 1999). These observations imply an upper limit of $2 \%$ on the rms amplitude of optical flux fluctuations on timescales from 0.1 to $2 \mathrm{hr}$ during the first day of the afterglow. After 1.6 days, the afterglow decline rate steepened, possibly because of the lateral expansion of a jet (Stanek et al. 1999; Harrison et al. 1999). Lateral expansion is expected to be important when the Lorentz factor decelerates to a value of order the inverse of the jet opening angle but could be neglected at earlier times (Rhoads 1997, 1999). In the following, we focus on the early stage of this afterglow ( $<1.6$ days), during which the observed afterglow radiation originates from a region much smaller than the jet opening angle. At this stage, the observed region behaves as if it is part of a spherically symmetric fireball and hence should be adequately described by our spherical expansion model. Our model also assumes that the unperturbed ambient gas has a uniform density. Chevalier \& Li (2000) studied wind interaction models for GRB afterglows and concluded that GRB 990510 can be better explained by a constantdensity medium than a wind density profile.

The equivalent $\gamma$-ray energy release of GRB 990510 for isotropic emission is $E=1.2 \times 10^{53}$ ergs, if the source redshift is $z=1.62$ (Wijers et al. 1999). We assume that the hydrodynamic energy release is comparable to this value and also adopt $n=1 \mathrm{~cm}^{-3}$. The afterglow emission peaks in the optical $\left(v_{\text {peak }}=5.1 \times 10^{14} \mathrm{~Hz}\right)$ at $T_{\text {obs }}=9.6 \mathrm{hr}$, when $\gamma_{b}=22$ and $R_{b}=3.9$ $\times 10^{17} \mathrm{~cm}$. Since most of the observational data from Stanek et al. (1999) is at frequencies $v>v_{\text {peak }}$, we compute the flux fluctuations in this regime. Our calculations indicate that flux fluctuations on timescales $\left(\tau_{1 / 2, \text { obs }}\right)$ between $0.3 \mathrm{and} 2 \mathrm{hr}$ correspond to density fluctuations on length scales, $r_{0}$, between $3.5 \times 10^{14}$ and $2.6 \times 10^{15} \mathrm{~cm}$, i.e., in the range $\sim 20-200 \mathrm{AU}$. Based on the observed upper limit on the amplitude of flux fluctuations in GRB 990510 we calculate the upper limit on $\xi_{0}^{1 / 2}$ as a function of $r_{0}$ in this range. The resulting constraints on the ISM inhomogeneities in the vicinity of the progenitor of GRB 990510 are illustrated by the solid line in Figure 5. The horizontally shaded region above this curve is forbidden, while the region below the curve is allowed, based on the afterglow data. We find that the amplitude of density fluctuations has to be lower than $\sim 10 \%$ on the length scale of $\sim 200$ AU and lower than unity on the $\sim 20$ AU scale.

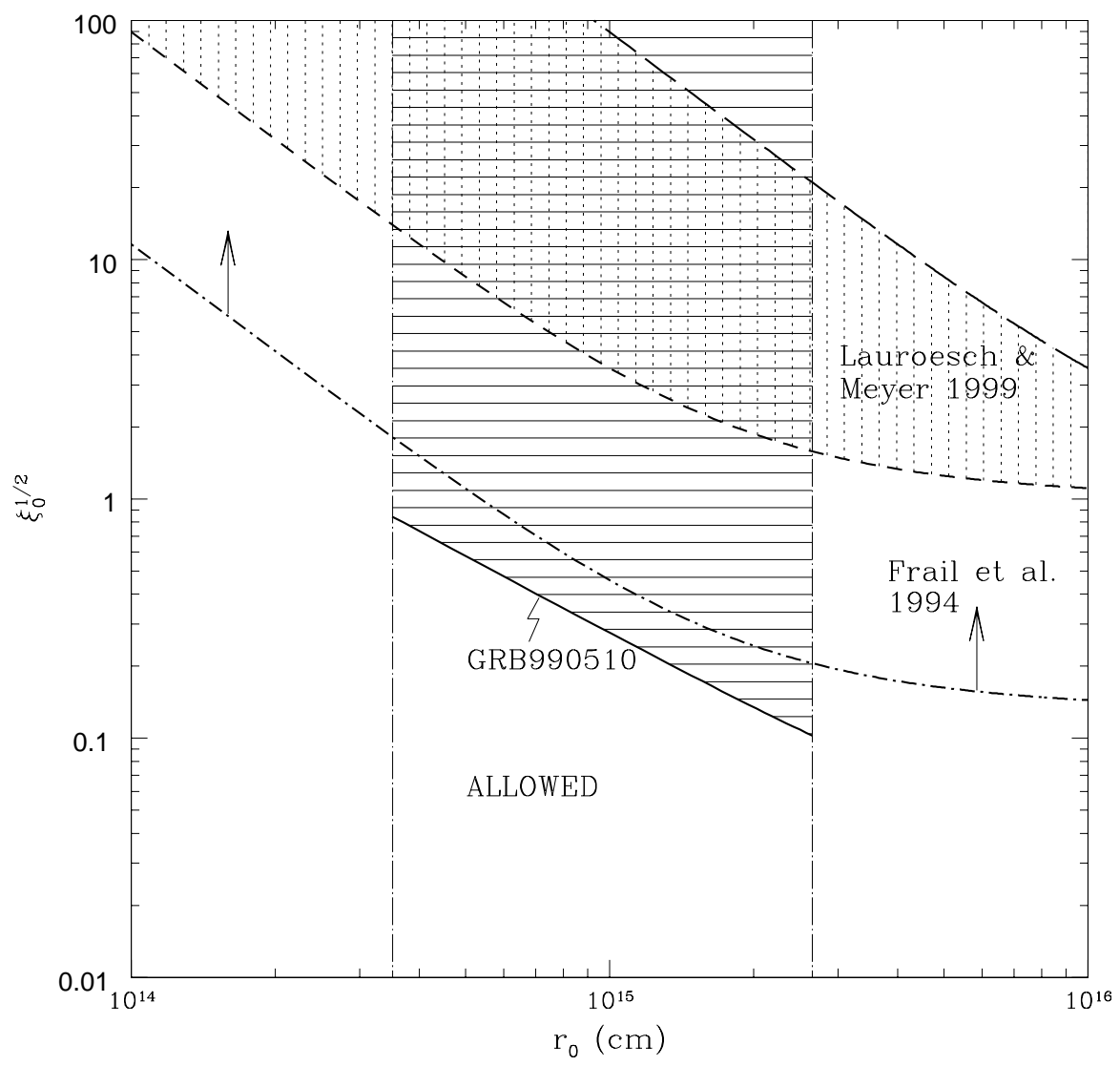

FIG. 5.- Constraints on the $\xi_{0}-r_{0}$ parameter space, for a density autocorrelation function of the form $\xi(r)=\xi_{0} \exp \left(-r / r_{0}\right)$. The solid curve describes the upper limit on $\xi_{0}^{1 / 2}$ for $r_{0}$ between $4 \times 10^{14}$ and $3 \times 10^{15} \mathrm{~cm}$ based on the upper limit on the amplitude of optical flux fluctuations in the GRB 990510 afterglow (Stanek et al. 1999). The upper horizontally shaded region is not allowed by the GRB 990510 data. The short-dashed curve and the long-dashed curve reflect constraints on the inhomogeneities in the local ISM, based on Lauroesch \& Meyer (1999), with the short-dashed curve corresponding to $\left\langle(\delta n / n)_{R}^{2}\right\rangle=1$ in spheres of radius $R=10^{2} \mathrm{AU}$ and the long-dashed curve corresponding to $\left\langle(\delta n / n)_{R}^{2}\right\rangle=1$ in spheres of radius $R=10^{3} \mathrm{AU}$. The vertically shaded region between these two curves describes intermediate length scales. The dot-dashed curve corresponds to $\left\langle(\delta n / n)_{R}^{2}\right\rangle^{1 / 2}=0.13$ in spheres of radius $R=10^{2} \mathrm{AU}$ and describes the lower limit on $\xi_{0}^{1 / 2}$ inferred from Frail et al. (1994). 
It is instructive to compare our derived constraints with observational data for density fluctuations on similar length scales in the ISM of the Milky Way. Structure on small scales was first inferred by Dieter, Welch, \& Romney (1976), using VLBI observations at $21 \mathrm{~cm}$ against the extragalactic source 3C 147. Diamond et al. (1989) and Davis, Diamond, \& Goss (1996) obtained similar results for more sources. Frail et al. (1994) detected temporal variations in the $21 \mathrm{~cm}$ absorption toward six high-velocity pulsars and inferred changes in the $\mathrm{H}$ I column density of $\sim 13 \%$ on projected scales of 5-100 AU. This sets a lower limit on the rms density contrast $\left(\delta n_{\mathrm{H}} / n_{\mathrm{H}}\right)$ of $\sim 13 \%$ in spheres of the above scale; the actual density contrast could be much larger because of partial cancellations between overdense and underdense regions along the line of sight to the pulsars. Lauroesch \& Meyer (1999) studied the small-scale ISM structure in atomic gas by observing the interstellar K I absorption line toward multiple star systems and inferred a hydrogen density contrast $\left(\delta n_{\mathrm{H}} / n_{\mathrm{H}}\right) \sim 1-2$ on the length scales of $10^{2}-10^{3} \mathrm{AU}$ (D. M. Meyer 1999, private communication). Small-scale density inhomogeneities were also inferred in molecular clouds (Marscher, Moore, \& Bania 1993; Moore \& Marscher 1995). All these studies indicate that structure is ubiquitous on scales of 10-10 ${ }^{3} \mathrm{AU}$ in the ISM (for a physical interpretation of the above results, see Heiles 1997). The observed structure might be caused in part by fluctuations in the ionization fraction or chemistry. However, for the purpose of putting our results in the context of these local ISM observations, we will assume that they relate to actual inhomogeneities in the gas density. In order to compare our results with the above data we need a relation between the autocorrelation function and the rms amplitude of density contrast in a region of a given size. For a spherical region of radius $R$, this relation is to a good approximation given by (Padmanabhan 1993, p. 200)

$$
\left\langle\left(\frac{\delta n}{n}\right)_{R}^{2}\right\rangle \cong \frac{1}{2 \pi^{2} V} \int_{0}^{R^{-1}} k^{3} \sigma_{k}^{2} \frac{d k}{k},
$$

where $V$ is the normalization volume and $\sigma_{k}^{2}$ is the power spectrum of the density fluctuation and is equal to the Fourier transform of the autocorrelation function:

$$
\sigma_{k}^{2}=V \int \xi(\boldsymbol{r}) e^{-i \boldsymbol{k} \cdot \boldsymbol{r}} d^{3} \boldsymbol{r}
$$

For the autocorrelation function in equation (32),

$$
\begin{aligned}
\sigma_{k}^{2} & =V \int_{0}^{\infty} d r \int_{0}^{\pi} d \theta \int_{0}^{2 \pi} d \phi \xi_{0} e^{-r / r_{0}} e^{-i k r \cos \theta^{2}} \sin \theta \\
& =4 \pi \xi_{0} V \int_{0}^{\infty} \frac{r}{k} e^{-r / r_{0}} \sin k r d r .
\end{aligned}
$$

Substituting this result into equation (43), we get

$$
\left\langle\left(\frac{\delta n}{n}\right)_{R}^{2}\right\rangle=\frac{2}{\pi} \xi_{0} \int_{0}^{\infty} r e^{-r / r_{0}} d r \int_{0}^{R^{-1}} k \sin k r d k=\frac{2}{\pi} \xi_{0}\left[\arctan \left(\frac{r_{0}}{R}\right)-\frac{R / r_{0}}{1+\left(R / r_{0}\right)^{2}}\right] .
$$

Based on observational constraints for $\left\langle(\delta n / n)_{R}^{2}\right\rangle$ on a given length scale $R$, we may now calculate from equation (47) the related value of $\xi_{0}^{1 / 2}$ for any assumed value of $r_{0}$.

The short-dashed curve in Figure 5 shows the constraint imposed by setting $\left\langle(\delta n / n)_{R}^{2}\right\rangle=1$ for $R=10^{2}$ AU, while the long-dashed curve corresponds to $\left\langle(\delta n / n)_{R}^{2}\right\rangle=1$ for $R=10^{3}$ AU. The vertically shaded region between these two curves describes intermediate length scales, referring to the range of constraints imposed by the observations of Lauroesch \& Meyer (1999). This entire region is well above the solid curve calculated from GRB 990510, implying that we should not have been surprised if flux fluctuations at a level of a few percent were detected in the optical afterglow of GRB 990510. The dot-dashed curve in this plot corresponds to setting $\left\langle(\delta n / n)_{R}^{2}\right\rangle^{1 / 2}=0.13$ for $R=10^{2} \mathrm{AU}$, which refers to the lower limit on the $\mathrm{H}$ I density fluctuations inferred by Frail et al. (1994). The allowed range of density fluctuations are above this curve. This constraint is close but still above the solid curve, i.e., in the region excluded by the variability data on GRB 990510. In summary, we find that if a GRB occurs in the ISM where the density perturbations have similar properties to those inferred by Lauroesch \& Meyer (1999) or Frail et al. (1994), then the resulting fluctuations in the afterglow flux should be detectable.

\section{CONCLUSIONS}

We have found that linear density fluctuations with $\delta n / n \lesssim 1$ on the length scale of $\sim 1-10^{3}$ AU could induce afterglow flux fluctuations with a fractional amplitude of up to $\sim 40 \%$ over timescales of tens of seconds in the X-rays, up to $\sim 30 \%$ over tens of minutes in the optical and up to $\sim 9 \%$ over days in the radio (see Table 3 ). These flux fluctuations average over the full range of density inhomogeneities within the emission region. For example, during the optical afterglow, the emission region occupies a rather large volume of $\sim\left(10^{4} \mathrm{AU}\right) \times\left(10^{3} \mathrm{AU}\right) \times\left(10^{3} \mathrm{AU}\right)$ (assuming $E_{52}=1, n_{1}=1$, and $\left.z=1\right)$, so inhomogeneities on scales $\ll 10^{3}$ AU would surely be ensemble averaged as long as their volume filling fraction is not too small.

At both extremes of high (X-ray) and low (radio) frequencies, the calculated variability might be contaminated by other effects. During the early period of the X-ray afterglow, the externally induced fluctuations we considered might be blended with variability associated with internal shells within the fireball that are still catching up with the decelerating blast wave and that were ignored in our analysis. At the opposite extreme of low radio frequencies, the flux might scintillate because of inhomogeneities in the local (Galactic) interstellar medium along the line of sight (Goodman 1997; Frail et al. 1997; Frail, Waxman, \& Kulkarni 2000; Waxman, Kulkarni, \& Frail 1998). In principle, the radio flux variability predicted by our model 
can be distinguished from variability due to scintillations, based on its different dependence on photon frequency, especially at high frequencies. However, it appears that the best spectral regime for observing the afterglow flux variability predicted in this paper is in between the X-ray and radio frequency windows, e.g., in the optical-infrared band.

Our calculation assumed spherical symmetry and should apply to the early expansion stages of a jet, as long as its Lorentz factor is larger than the inverse of its opening angle (Rhoads 1997, 1999; Sari et al. 1999). Our treatment could be extended in the future to describe the lateral expansion of a jet at later times, the possible existence of a power-law density profile as for a precursor wind from the GRB progenitor (Chevalier \& Li 2000), and the possible effects of nonlinear clumps of density (Dermer \& Mitman 1999).

The application of our simple model to the early optical afterglow of GRB 990510 (Stanek et al. 1999) provides already interesting upper limits on the density fluctuations on scales of 20-200 AU around the source. These limits are lower than the observed fluctuation amplitude on similar scales in the local interstellar medium (Fig. 5). If these local measurements apply to interstellar turbulence in high-redshift galaxies, then optical monitoring of future afterglows should reveal flux fluctuations at the level of a few percent or higher on a timescale of less than $1 \mathrm{hr}$.

We thank Bruce Draine, Dale Frail, and David Meyer for useful discussions about the detection of density fluctuations in the ISM. This work was supported in part by NASA grants NAG5-7039 and NAG5-7768.

Blandford, R. D., \& McKee, C. F. 1976, Phys. Fluids, 19, 1130

Chevalier, R. A., \& Li, Z. Y. 2000, ApJ, in press

Davis, R. J., Diamond, P. J., \& Goss, W. M. 1996, MNRAS, 283, 1105

Dermer, C., \& Mitman, K. 1999, ApJ, 513, L5

Diamond, P. J., Goss, W. M., Romney, J. D., Booth, R. S., Kalberla, P. N. W., \& Mebold, U. 1989, ApJ, 347, 302

Dieter, N. H., Welch, W. J., \& Romney, J. D. 1976, ApJ, 206, L113

Frail, D. A., Kulkarni, S. R., Nicastro, L., Feroci, M., \& Taylor, G. B. 1997, Nature, 389, 261

Frail, D. A., Waxman, E., \& Kulkarni, S. R. 2000, ApJ, submitted

Frail, D. A., Weisberg, J. M., Cordes, J. M., \& Mathers, C. 1994, ApJ, 436, 144

Goodman, J. 1997, NewA, 2, 449

Granot, J., Piran, T., \& Sari, R. 1999, ApJ, 513, 679

Harrison, F. A., et al. 1999, ApJ, 523, L121

Heiles, C. 1997, ApJ, 481, 193

Hjorth, J., et al. 1999, GCN Circ. 320 (http://gcn.gsfc.nasa.gov/gcn3/ 320.gcn3)

Katz, J. I. 1994, ApJ, 422, 248

Lauroesch, J. T., \& Meyer, D. M. 1999, ApJ, 519, L181

Marscher, A. P., Moore, E. M., \& Bania, T. M. 1993, ApJ, 419, L101

Mészáros, P. \& Rees, M. J. 1993, ApJ, 405, 278 -. 1997, ApJ, 476, 232

\section{REFERENCES}

Moore, E. M., \& Marscher, A. P. 1995, ApJ, 452, 671

Paczyński, B., \& Rhoads, J. E. 1993, ApJ, 418, L5

Padmanabhan, T. 1993, Structure Formation in the Universe (Cambridge: Cambridge Univ. Press)

Piran, T. 1999, Phys. Rep., 314, 575

Reif, F. 1965, Fundamentals of Statistical and Thermal Physics (New York: McGraw-Hill)

Rhoads, J. E. 1997, ApJ, 487, L1 1999, ApJ, 525, 737

Rybicki, G. B., \& Lightman, A. P. 1979, Radiative Processes in Astrophysics (New York: Wiley)

Sari, R., Piran, T., \& Halpern, J. P. 1999, ApJ, 519, L17

Sari, R., Piran, T., \& Narayan, R. 1998, ApJ, 497, L17

Stanek, K. Z., Garnavich, P. M., Kaluzny, J., Pych, W., \& Thompson, I. 1999, ApJ, 522, L39

Waxman, E. 1997a, ApJ, 485, L5 . 1997b, ApJ, 489, L33

. 1997c, ApJ, 491, L19

Waxman, E., Kulkarni, S. R., \& Frail, D. A. 1998, ApJ, 497, 288

Wijers, R. A. M. J., \& Galama, T. J. 1999, ApJ, 523, 177

Wijers, R. A. M. J., Rees, M. J., \& Mészáros, P. 1997, MNRAS, 288, L51

Wijers, R. A. M. J., et al. 1999, ApJ, 523, L33 